Website: ijetms.in Issue:3, Volume No.4, May-2020 DOI: 10.46647/ijetms.2020.v04i03.002

\title{
Crop Analysis Using Image Processing
}

\author{
Srujana. $\mathrm{V}^{1}$,Chaithanya.P ${ }^{2}$,Dr.B.Ramesh ${ }^{3}$,Vanga Mahesh ${ }^{4}$,Manorajan Soora ${ }^{5}$ \\ ${ }^{1}$ Asst.professor, Kamala Institute of Technology \& Science (Huzurabad) \\ ${ }^{2}$ Asst. professor, Kamala Institute of Technology \& Science (Huzurabad) \\ ${ }^{3}$ Professor, Kamala Institute of Technology \& Science (Huzurabad) \\ ${ }^{4}$ Asst.professor, Kamala Institute of Technology \& Science (Huzurabad) \\ ${ }^{5}$ Asst.professor, Warangal Institute of Technology \&Science(Warangal)

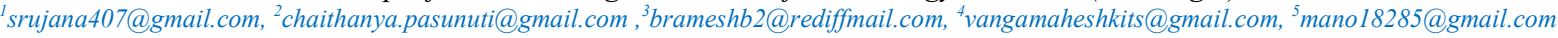

Abstract - To detect the uniqueness and quantities of agriculture product images a new method is proposed using MATLAB software .In this paper we propose a method to increase the contrast level of a image with exponential low pass filter and histogram equalization technique. Next by using region props function we extract the binary features of the image, and then we calculated the number of targets in gray level image. This method can be easily applied in modern agriculture.

Keywords-Histogram equalization, bwlabel, regionprop, bwboudaries

\section{Introduction}

With the advent of the era of high-speed information technology, the application of computer image processing is more extensive. Traditional data based on limited measurements can't meet the requirements of precision agriculture in terms of accuracy and convenience. Besides, traditional manual management methods can't obtain agricultural information timely and efficiently. Taking above conditions into consideration, this paper focuses on agricultural products, and uses computer image processing and analysis technology, aiming at automatically identifying and understanding images to detect the characteristics and quantities of agricultural products.

Miller and Delwiche developed a laboratory machine vision system to detect and identify surface defects like scar, cuts, bruise etc on fresh market peaches [1]. Image analysis algorithms were developed for segmenting defect regions in the peach images, and identify specific type of defect. To measure rice canopy Cascady used machine vision technology [2]. To identify crops and weeds Critten used Fourier analysis [3], but techniques by which shade is converted into shape are inherently unreliable.

\section{Image Enhancement:}

Image Enhancement plays a significant role in improving image quality [4]. But image enhancement is a paradoxical process. On the one hand, image enhancement technology hopes to enhance edges and remove noise. On the other hand, removing noise will blur the edges to a certain extent, while enhancing edges will increase noise meanwhile. Therefore, it is necessary to balance the two when considering image enhancement. By using image enhancement noise components like salt and pepper in the image are eliminated.

Based on principle of operation image enhancement can be divided in to two types namely special domain method and Frequency domain method. Spatial domain method operates on pixel values .Frequency domain method operates on frequency transformation.

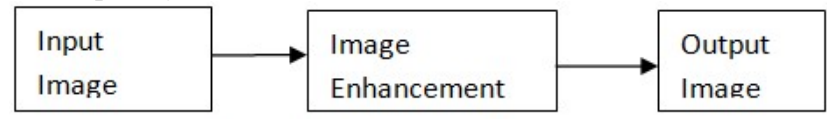

Fig:1 Block diagram of image enhancement

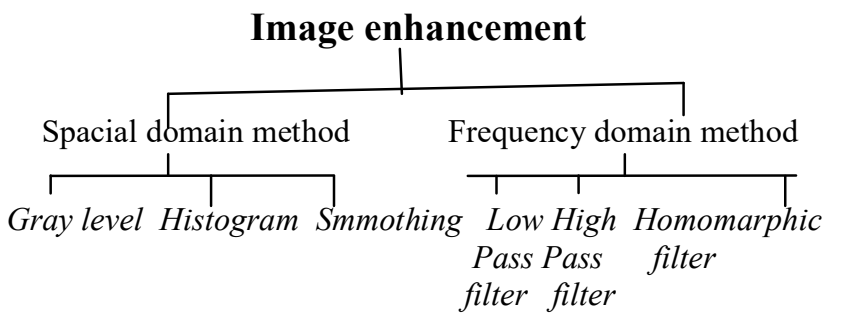

Fig:2 Types of Image enhancement

\subsection{Gray scale transformation:}

Image enhancement is done through gray level Transformation .Gray level transformation is done in three ways like power law, linear and logarithmic.

\section{Linear transformation}

This method includes simple identity and negative transformation. Identity transition shown by a straight line. In this conversion, each value of the input image is directly mapped to each other value of output image. That results in the same input image and output image. So , this method also as called identity transformation. It has been shown below:

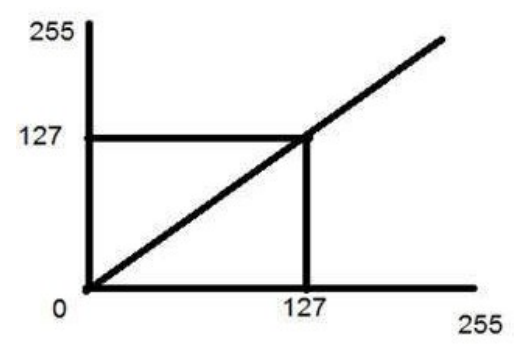

Fig:3 Transition diagram 
Website: ijetms.in Issue:3, Volume No.4, May-2020 DOI: 10.46647/ijetms.2020.v04i03.002

The negative transformation is a second linear transformation, which is invert of identity transformation.

In this transformation, each value of the input image is subtracted from the L-1 and mapped onto the output image.

In this transformation the following transition has been done.

$$
\mathrm{S}=(\mathrm{L}-1)-\mathrm{r}
$$

The numbers of levels (L) in the input image are 256. By Putting 256 in the equation 1 , we get $\mathrm{s}=255$-r.In this transition each pixel value is subtracted by 255 and the result image has been shown Fig5. In the output image, the lighter pixels become dark and the darker pixels become light. And it results in negative image.

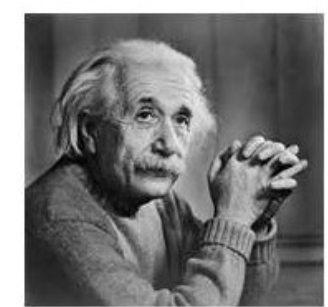

Fig:4 Input image

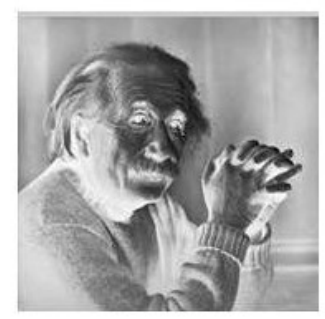

Fig: 5.Output image

\section{Logarithmic transformation:}

Logarithmic transformation is divided into two types Log transformation and inverse log transformation.

For logarithmic transformation the formula is

$$
\mathrm{S}=\mathrm{clog}(\mathrm{r}+1)
$$

Where,

$\mathrm{S}=$ pixel values for output

$\mathrm{R}=$ pixel values for input

$\mathrm{c}=$ constant.

In log transformation, dark pixels are expanded as compared to higher pixel values and lighter pixels are compressed.

\section{Power-Law transformation:}

Power law transformation includes nth power and nth root transformation. Power law transformation is used for enhancing images for different display devices. The gamma values are different for different display devices.

For power law transformation the formula is

$$
\begin{gathered}
\mathrm{S}=\mathrm{cr}^{\wedge} \gamma \ldots \ldots(3) \\
\text { Where } \\
\mathrm{S}=\text { output pixel value } \\
\mathrm{R}=\text { input pixel value } \\
\mathrm{c} \text { and } \gamma \text { are constants }
\end{gathered}
$$

For various values of $\gamma$ different levels of enhancement is done in the image.

\subsection{Histogram equalization:}

To improve the contrast levels in the image Histogram Equalization is used. It is a special enhancement technique. By spreading out the most frequent intensity values contrast level of the image is improved. The pixel values will range from 0 to 255 .

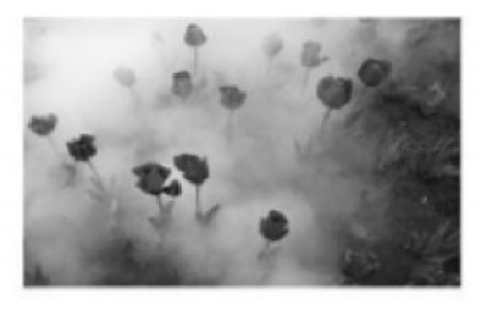

Fig6: Flower image

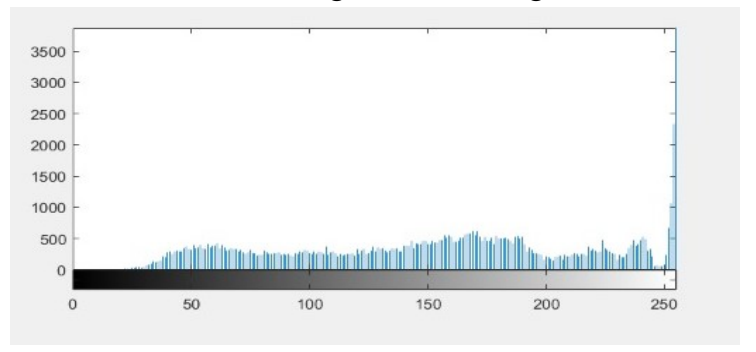

Fig7: Histogram of values of flower image

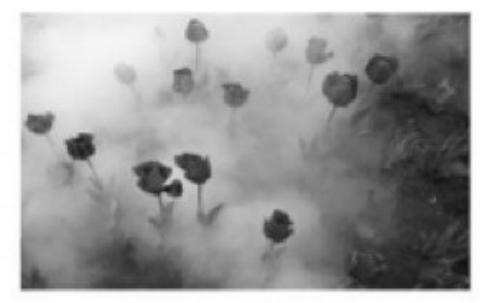

Fig.8: histogram Equalization image

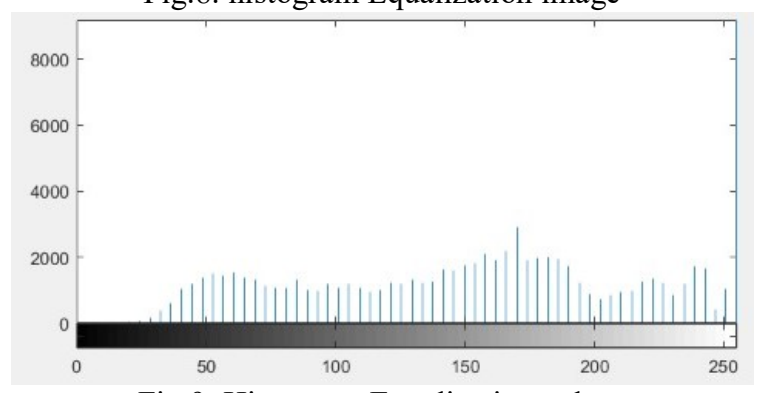

Fig.9: Histogram Equalization values

In Histogram equalization first we calculate the probability mass function and cumulative distribution function for adjusting the pixel values in an image to enhance the contrast level of the image. The histogram of an image will have close to normal distribution where as histogram equalization is uniform distribution.

\subsection{Low pass filter:}

Low pass filter allows only low frequencies. The energy of the image mostly concentrated in the low and 
Website: ijetms.in Issue:3, Volume No.4, May-2020 DOI: 10.46647/ijetms.2020.v04i03.002

medium frequencies and noise of the image corresponds to high frequencies.

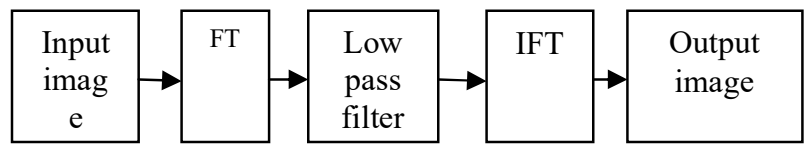

Hence low pass filter does not allow high frequencies so some of the noise components of the image are eliminated by low pass filter.

\section{Image segmentation:}

The process of partitioning a digital image into sets of pixels or image objects is known as image segmentation. We can easily locate the objects and boundaries in the image using pixel values. Image segmentation place a major rule in this paper.

There are three approaches in image segmentation

1. Region based approach

2. Boundary approach

3. Edge approach

\subsection{Region based segmentation approach:}

A group of connected pixels with similar properties in a image are called Regions. In the region based segmentation approach, each pixel is assigned to a particular object or region. Segmentation algorithms based on region easily manageable and more immune to noise compared to edge detection methods. In region based methods, partition an image into regions that are close according to a set of predefined criteria.

In the region based segmentation, pixels corresponding to an object are grouped together and marked. This method also requires the use of appropriate thresholding techniques. The important principles are usefulness similarity and spatial proximity.

\subsection{Segmentation based on Boundary:}

In order to overcome some of the limitations of region based methods, boundary based methods are often used to look for explicit or implicit boundaries between regions. The two most commonly used boundary based methods are known as ridge detection and edge detection.

\subsection{Segmentation based on Edge:}

In edge based segmentation technique edges in an image are concluded to represent object boundaries, and handled down to recognize these objects. To observe the edges in an image is to focus for places in the image where the intensity changes proximity. There are two main edge based segmentation methods gray histogram and gradient based method.

\subsection{Image extraction:}

To obtain image segmentation we use im $2 \mathrm{bw}$ function. im 2 bw function is used to convert gray level image to binary image. To display the boundaries of the image we use the bwboundaries function. This function returns the row and column coordinates of border pixels of all the objects in an image.

\section{Flow chart:}

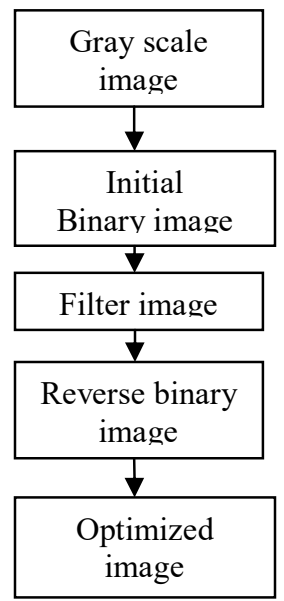

Step1: First we taken the grayscale image

Gary scale image: a grayscale or grayscale image is one in which the value of each pixel is a single sample representing only an amount of light.

Step2: Convert grayscale image to binary image

Binary image: A binary image is one that consists of pixels that can have one of exactly two colors, usually black and white.

Step3: Remove the noise using median filter.

Step4: Applied bwboundary function to the filtered image to identify boundaries in the image

Step5: Applied bwlabel function to the boundary image Step6: Appllied cmap function to the labeld image to identify objects in the image with colours

Step7: Applied regionprop function to get maximum area, minimum area, centroid etc for the Image.

Step8: Applied the count function to calculate the required objects in the image

Results:

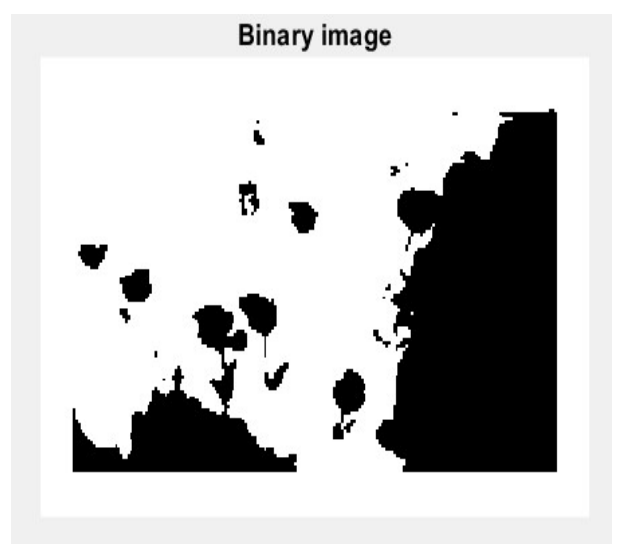


Website: ijetms.in Issue:3, Volume No.4, May-2020 DOI: 10.46647/ijetms.2020.v04i03.002

Binary image

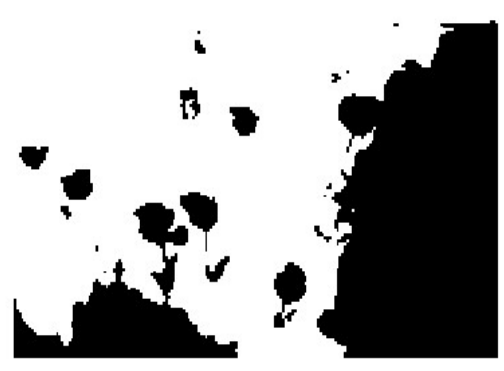

Filter image

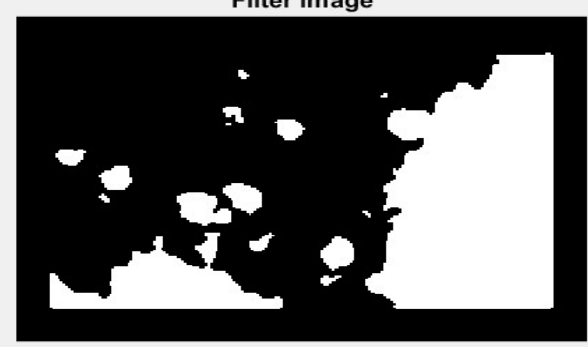

BW Boudaries image

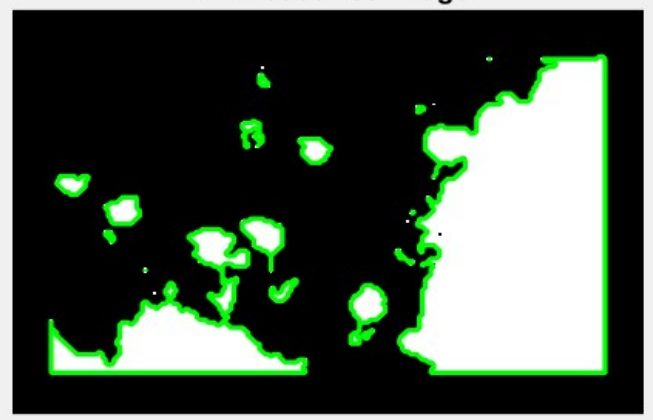

Filter image

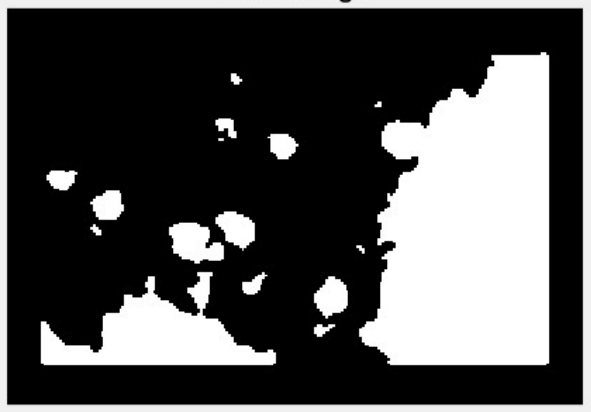

label image
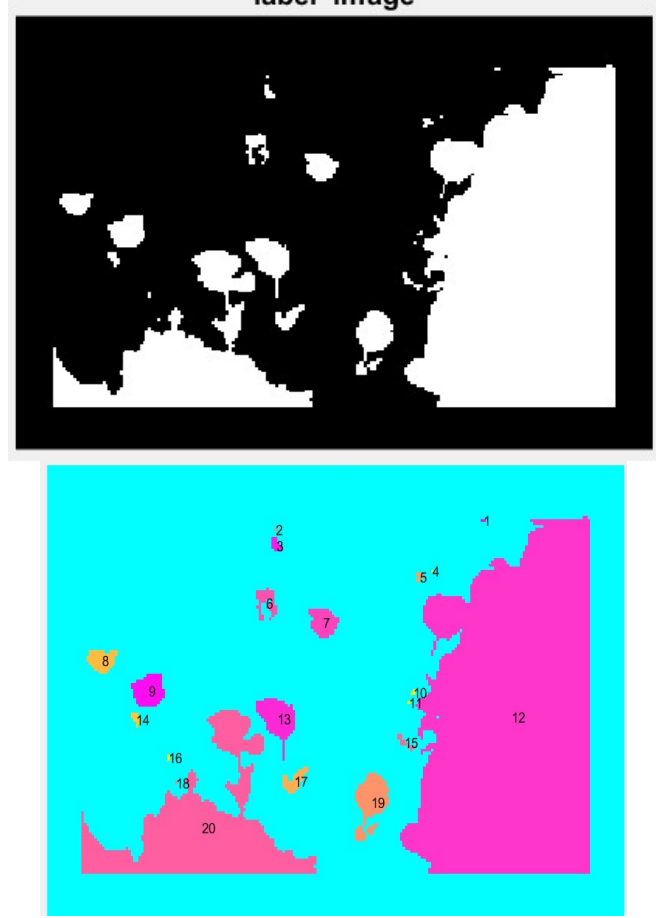

Fig.12.Mark sucessive areas

We choose the regionprops function to extract the followingbinary features for each target in the image. 
Website: ijetms.in Issue:3, Volume No.4, May-2020 DOI: 10.46647/ijetms.2020.v04i03.002

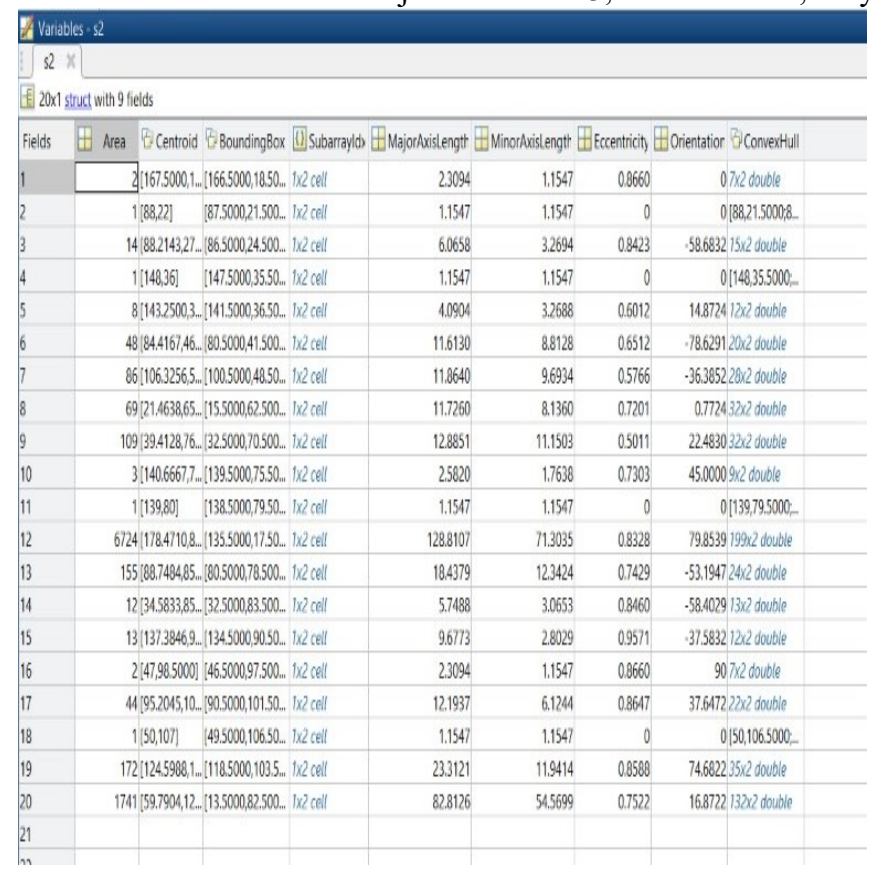

[5] Mahesh, B., and K. Shyam Sunder Reddy. "Router Aided Congestion Control Techniques." Second International Conference on Information Systems and Technology.

\section{Conclusion}

This paper proposes a method to detect the quantities and characteristics of agriculture products. The combination of image enhancement and image extraction is ahieved, and the contrast and gray dynamic range of the image are improved to a certain extent. We have access to agriculture information more faster.

\section{References}

[1] MILLERBK, DELWICHE M J. "Peach defect detection with machine vision" [J].Transactions of the ASAE, 1991, 34 (6): 2588-2597.

[2] Casady $W W$, Singh N, Costello $T A$. "Machine Vision for Measurement of Rice Canopy Dimensions " $[\mathrm{J}]$. Transactions of the ASAE, 1996, 39 (5):1891-1898.

[3] Critten D L. "Fourier based techniques for the identification of plants and weeds" [J]. Journal of agricultural engineering research, 1996, 64 (2): 149-154.

[4] Rahman Z, Jobson D J, Woodell G A." Retinex processing for automatic image enhancement" $[\mathrm{J}]$. Journal of Electronic imaging, 2004, 13 (1): 100-111. Rahman Z, Jobson D J, Woodell G A. Retinex processing for automatic image enhancement [J]. Journal of Electronic imaging, 2004, 13 (1): 100-111. 